\title{
Parto tras la transferencia de embriones generados por fecundación de ovocitos vitrificados. Comunicación de un caso
}

\author{
RICARDO POMMER ${ }^{1}$, FRANCISCA JERIA ${ }^{1,2}$, BEGOÑA ARGUELLO ${ }^{1,2}$, \\ SONIA VILLA ${ }^{1}$, JOSÉ BALMACEDA ${ }^{1}$, JUAN ENRIQUE SCHWARZE ${ }^{1,3, a}$
}

Unidad de Medicina Reproductiva de Clínica Monteblanco, Santiago, Chile. 2Embrióloga.

${ }^{3}$ Departamento Clínico de Obstetricia y Ginecología de la Universidad de Santiago de Chile. ${ }^{a} \mathrm{MSc}$.

Recibido el 30 de julio de 2010, aceptado el 26 de mayo de 2011.

Correspondencia a: Dr. Juan Enrique Schwarze UMR Clínica Monteblanco. Camino a Farellones, KM 4 Lo Barnechea, Santiago Chile. E-mail: jeschwarze@mac. com

\section{Live birth after the transfer of embryos obtained by fertilization of vitrified oocytes. Report of one case}

We report the first successful live birth after the transfer of embryos obtained by fertilization of vitrified oocytes. A couple with primary infertility due to teratozoospermy and chronic anovulation decided to undergo assisted reproductive technology after 4 failed cycles of superovulation and intrauterine insemination. The woman underwent a standard luteal phase agonist protocol, with controlled ovarian hyper stimulation with daily 150 IU recombinant gonadotropin and 75 IU urinary gonadotropin for 12 days. Due to the high risk of ovarian hyper stimulation syndrome the couple was advised to delay embryo transfer. Eighteen mature oocytes were recovered, eight were vitrified and 10 fertilized and cryopreserved as pro-nuclei. Two months later, four vitrified oocytes were thawed, and three morphologically-normal embryos were transferred to an estrogen-progesterone-primed uterus, obtaining a triple clinical pregnancy. The pregnancy was uneventful until 32 weeks of amenorrhea, when preterm delivery started. After inducing lung maturity, a cesarean section was performed and three healthy fetuses were delivered. Nowadays, the babies are two and a half years old, and have had a normal psycho-motor development.

(Rev Med Chile 2011; 139: 920-923).

Key words: Embryo, mammalian; Fertilization; Fetus; Oocytes.
L a criopreservación embrionaria en las distintas etapas del desarrollo preimplantacional es una técnica utilizada rutinariamente en los centros de medicina reproductiva de todo el mundo. Al permitir la transferencia secuencial de los embriones, permite la fecundación de un mayor número de ovocitos, obteniendo así más embriones, aumentando la posibilidad de que al menos uno de éstos sea normal y al implantar genere un embarazo. De este modo se evita la transferencia de un mayor número de embriones que se asocia a un aumento del riesgo de multigestación y de las complicaciones asociadas a la prematurez ${ }^{1}$.

La acumulación de embriones criopreservados presenta una seria complicación para los centros de reproducción asistida. Asimismo, algunas parejas tienen conflictos del punto de vista ético con esta técnica. De este modo, la criopreservación de ovocitos representa una alternativa atractiva. Sin embargo, es una tecnología que presenta mucho más dificultades técnicas que la criopreservación embrionaria. Las dificultades técnicas se deben al gran tamaño de los ovocitos y su alto contenido de agua. El agua citoplasmática debe ser removida casi en su totalidad para evitar la formación de cristales de hielo, capaces de dañar mecánicamente las estructuras celulares, por ejemplo, el citoesqueleto, que en el caso de los ovocitos maduros se puede traducir en daño del huso meiótico, que 
mantiene los cromosomas alineados en la placa metafásica, lo que puede causar aneuploidía en las células hijas ${ }^{3}$.

Hay dos estrategias para criopreservar: la criopreservación lenta, en que la temperatura disminuye lentamente, comúnmente a una tasa de $-0,3^{\circ} \mathrm{C} / \mathrm{min}$; y la vitrificación, un tipo de criopreservación ultrarrápido, con inmersión directa en nitrógeno líquido ${ }^{4}$.

A partir del primer embarazo en 1983 por transferencia de embriones descongelados, la congelación lenta ha sido la estrategia más utilizada, dando muy buenos resultados en el caso de criopreservación embrionaria. En el caso de ovocitos, ningún grupo ha logrado brindar resultados adecuados y reproducibles en términos de sobrevida tras la descongelación, fecundación y embarazo.

En los últimos años, se han conseguido resultados satisfactorios con la vitrificación de ovocitos, publicándose excelentes tasas de sobrevida, fecundación y embarazo ${ }^{3,5,6}$. En el presente reporte presentamos el primer caso de embarazo en Chile tras la transferencia de embriones producto de la fecundación de ovocitos vitrificados.

\section{Caso clínico}

La pareja consultó con una historia de infertilidad primaria de 2 años. Ella de 36 años y su marido de 37 años. Dentro del estudio inicial se encontró oligoastenozoospermia moderada. Ella además presentaba anovulación secundaria a un síndrome de ovario poliquístico. Luego de intentar 4 ciclos de superovulación con inseminación intrauterina sin éxito, se decidió optar por un ciclo de alta complejidad en el 2007, para el cual la pareja dio su consentimiento, luego de responder y resolver todas sus dudas.

\section{Hiperestimulación ovárica controlada}

Luego de suprimir la secreción endógena de gonadotrofinas mediante la administración de 0,5 mg sc diarios de acetato de leuprolida por 7 días, evidenciado ecográficamente por la ausencia de folículos ováricos de diámetro $\geq 10 \mathrm{~mm}$, se estimuló el desarrollo polifolicular con la administración diaria de 150 IU sc de folitropina beta más 75 IU im de gonadotrofina menopáusica humana. El día 12 de estimulación, se observaron 22 folículos de $\geq 15 \mathrm{~mm}$ de diámetro, por lo cual se indicó
10.000 UI de HCG y 36 horas después, se realizó la culdocentesis bajo anestesia general.

Considerando el gran número de folículos y la concentración de estradiol plasmático $(27.793,5$ $\mathrm{pg} / \mathrm{ml}$ ) se aconsejó transferir los embriones en forma diferida, para disminuir el riesgo de desarrollar un síndrome de hiperestimulación ovárica. La paciente presentó un cuadro leve, que evolucionó favorablemente con medidas conservadoras, resolviéndose completamente en dos semanas.

\section{Vitrificación}

El protocolo utilizado fue publicado por Kuwayama en el $2005^{6}$. Según este protocolo los ovocitos fueron expuestos gradualmente a una solución de equilibrio con $7,5 \%$ de EG y 7,5\% de DMSO, seguido por una rápido exposición a la solución de vitrificación, con $15 \%$ de EG, $15 \%$ de DMSO y $0,5 \mathrm{M}$ sacarosa. Tras 1 minuto de exposición a esta solución, hasta 2 ovocitos fueron cargados en un contenedor con $0,1 \mu \mathrm{l}$ de volumen de carga y sumergidos rapidamente en nitrógeno líquido. Una vez dentro del nitrógeno, se cubrió con una tapa protectora. Finalmente, los contenedores fueron almacenados en un tanque de almacenamiento con nitrógeno líquido.

\section{Preparación endometrial}

Dos meses más tarde, se preparó el endometrio para la transferencia embrionaria. La preparación fue mediante la administración creciente de estradiol oral hasta conseguir un grosor endometrial bajo visión ultrasonográfica de $9 \mathrm{~mm}$. En ese momento se agregó $100 \mathrm{mg}$ im diarios de progesterona. El suplemento exógeno de estradiol y progesterona duró hasta completar el primer trimestre de embarazo, cuando la placenta comienza la síntesis en cantidad adecuada de estos esteroides.

\section{Desvitrificación y transferencia}

Los contenedores desvitrificados fueron traspasados desde el tanque de almacenamiento a una caja de polistireno con nitrógeno líquido. Luego el extremo se sumergió por 1 minuto en una solución de descongelación con sacarosa $1,0 \mathrm{M}$ a $37^{\circ} \mathrm{C}$. A continuación, los ovocitos fueron expuestos por 3 minutos a una solución de sacarosa $0,5 \mathrm{M}$, seguido de 2 pasos por 5 minutos por una solución de lavado. Finalmente, los ovocitos fueron traspasados a una cápsula con medio de cultivo equilibrado e incubados hasta su microinyección, 2 horas des- 
pués. Los embriones obtenidos fueron cultivados hasta su transferencia, en estadio de ocho células, transvaginal con un catéter ultrasuave, bajo visión ecográfica.

\section{Resultados}

Dieciocho de los 25 ovocitos recuperados estaban en metafase II. De estos 18 ovocitos maduros, 8 fueron vitrificados y 10 fueron fertilizados y vitrificados en estadío de pronúcleo. Una vez preparado el endometrio y tras discutir las opciones con la paciente, se decidió desvitrificar cuatro ovocitos.

Los cuatro ovocitos desvitrificados sobrevivieron, presentando morfología normal. Así mismo, los cuatro ovocitos fecundaron tras inyección intracitoplasmática de espermatozoides, y continuaron su desarrollo in vitro. Tres de los embriones, que presentaban buena morfología, fueron transferidos al tercer día de cultivo. El cuarto embrión, que presentaba alteraciones morfológicas, detuvo su desarrollo posteriormente.

Doce días después de la transferencia embrionaria, la medición de HCG fue 1.436,8 mUI/mL. Mediante ecografía transvaginal se evidenció la presencia de tres sacos gestacionales, el interior de cada uno se identificó un embrión con actividad cardiaca normal. La paciente no presentó complicaciones durante su embarazo, hasta que comenzó a presentar contracciones uterinas dolorosas a las 32 semanas de amenorrea. Luego de la administración profiláctica de corticoides para inducir maduración pulmonar fetal, se interrumpió por cesárea, naciendo tres neonatos vivo que evolucionaron favorablemente. Los pesos de los neonatos fueron 1.605 gramo, 1.505 gramos y 1.095 gramos. Los puntajes de Apgar al minuto y a los cinco minutos fueron $9 / 10,9 / 10 \mathrm{y}$ 8/9, respectivamente. Estuvieron hospitalizados veintinueve días para ganar peso, evolucionando sin complicaciones médicas de ningún tipo. En la actualidad tienen más de 3 años y todos su desarrollo psicomotor ha sido normal de acuerdo a la evaluación pediátrica habitual.

\section{Discusión}

Desde que se describió la criopreservación embrionaria, su aplicación ha crecido signifi- cativamente en el mundo, llevando hoy en día a la necesidad de crear nuevas estrategias que disminuyan el almacenamiento de embriones, ya sea recuperando menos ovocitos o limitando el número de ovocitos a fecundar. La posibilidad de criopreservar ovocitos permite limitar el número de embriones criopreservados, y ofrece una alternativa a aquellas parejas que rechazan la criopreservación embrionaria ${ }^{7}$.

Asimismo, la vitrificación de ovocitos no sólo es una alternativa de tratamiento para parejas infértiles, sino que abre una valiosa oportunidad a mujeres jóvenes afectadas por cáncer para conservar su potencial reproductivo ${ }^{5,8,9}$. Otro grupo de mujeres beneficiadas con esta técnica son las que han decidido postergar su maternidad debido a su desarrollo profesional y/o académico, enfrentándose al dilema del deterioro de su función ovárica y a la drástica disminución de su potencial reproductivo con la edad ${ }^{10}$.

En los últimos años, tras muchos esfuerzos por lograr buenos resultados, se han logrado las condiciones necesarias para contar con protocolos de vitrificación ovocitaria, que han entregado resultados homogéneos y reproducibles, con tasas de embarazo similares a las obtenidas en ciclos en fresco?.

El caso reportado constituye una muestra de que esta técnica ha sido implementada exitosamente en nuestro país, como una forma de evitar la criopreservación embrionaria o como una forma de preservar fertilidad.

\section{Referencias}

1. Zegers-Hochschild F. Attitudes towards reproduction in Latin America. Teachings from the use of modern reproductive technologies. Hum Reprod Update 1999 a; 5: 21-5.

2. Liebermann J, Dietl J, Vanderzwalmen P, Tucker MJ. Recent developments in human oocyte, embryo and blastocyst vitrification: where are we now? Reprod Biomed Online 2003 b; 7: 623-33.

3. Wininger JD, Kort HI. Cryopreservation of immature and mature human oocytes. Semin Reprod Med 2002 c; 20: 45-9.

4. Kim TJ, Laufer LR, Hong SW. Vitrification of oocytes produces high pregnancy rates when carried out in fertile women. Fertil Steri 2010 d; 93: 467-74.

5. Kuwayama M, Vajta G, Kato O, Leibo SP. Highly efficient 
Fecundación de ovocitos vitrificados - R. Pommer et al

vitrification method for cryopreservation of human oocytes. Reprod Biomed Online 2005 c; 11: 300-8.

6. Tao T, Zhang W, Del Valle A. Human oocyte cryopreservation. Curr Opin Obstet Gynecol 2009 d; 21: 247-52.

7. Domingo J, Ayllón Y, Domingo S, Cobo A, Crespo J, Pellicer A. New approaches to female fertility preservation. Clin Transl Oncol 2009 e; 11: 154-9.
8. Grifo JA, Noyes N. Delivery rate using cryopreserved oocytes is comparable to conventional in vitro fertilization using fresh oocytes: potential fertility preservation for female cancer patients. Fertil Steril 2010 f; 93: 391-6.

9. Homburg R, van der Veen F, Silber SJ. Oocyte vitrification-women's emancipation set in stone. Fertil Steril. 2009 e; 91: 1319-20. 